\title{
Conhecimento e atitudes de usuários com Diabetes Mellitus em uma unidade de ambulatório especializada
}

\author{
Knowledge and attitudes of users with Diabetes Mellitus on a specialized outpatient clinic \\ Conocimiento y actitudes de los usuarios con Diabetes Mellitus en una unidad ambulatoria \\ especializada
}

Ivânia Maria dos Santos ${ }^{1 *}$, Evylene Adlla Cavalcanti Lima ${ }^{1}$, Juliana de Oliveira Pimentel ${ }^{1}$, Isabella Joyce Silva de Almeida², Valesca Patriota de Souza ${ }^{1}$.

\section{RESUMO}

Objetivo: Caracterizar os usuários com Diabetes Mellitus segundo as variáveis sociodemográficas e clínicas e avaliar o conhecimento e atitude em relação à doença. Métodos: Trata-se de um estudo transversal de abordagem quantitativa, realizado com 173 usuários com Diabetes Mellitus cadastrados no Centro de Diagnósticos do Coração e Controle do Diabetes, no município de Vitória de Santo Antão, Pernambuco. Foi utilizado na coleta de dados um roteiro sistematizado para obter as variáveis sociodemográficas e clínicas, bem como os instrumentos de coleta: o Diabetes Knowledge Questionnaire para avaliar o conhecimento e o Diabetes Attitude Questionnaire para avaliar a atitude. Resultados: A população caracterizou-se por adultos e idosos, com renda de até um salário mínimo. Quanto ao conhecimento, 65,3\% dos usuários apresentam escores inferiores a oito pontos, o que evidencia conhecimento insatisfatório em relação a doença. Em relação às atitudes, constatou-se que a maioria $(98,3 \%)$ dos usuários apresentou escores inferiores a 70 pontos, indicando dificuldades no enfrentamento da doença. Conclusão: Conclui-se que o conhecimento e a atitude ainda são incipientes, emergindo a necessidade de implantação de programas de educação em saúde.

Palavras-chave: Diabetes Mellitus, Conhecimento, Atitude frente à saúde.

\section{ABSTRACT}

Objective: To characterize users with Diabetes Mellitus according to sociodemographic and clinical variables and to assess knowledge and attitude towards the disease. Methods: This is a cross-sectional study with a quantitative approach, carried out with 173 users with Diabetes Mellitus registered at the Heart Diagnosis and Diabetes Control Center, in the city of Vitória de Santo Antão, Pernambuco. In the data collection, a systematic script was used to obtain the sociodemographic and clinical variables, as well as the collection instruments: The Diabetes Knowledge Questionnaire to assess knowledge and the Diabetes Attitude Questionnaire to assess attitude. Results: The population was characterized by adults and the elderly, with an income of up to one minimum wage. Regarding knowledge, $65.3 \%$ of users had scores below eight points, which shows unsatisfactory knowledge of the disease. The majority $(98.3 \%)$ of users had attitude scores below 70 points, denoting difficulties in coping with the disease. Conclusion: It is concluded that the knowledge and attitude is still incipient, emerging the need to implement health education programs.

Key words: Diabetes Mellitus, Knowledge, Attitude to health.

\section{RESUMEN}

Objetivo: Caracterizar a los usuarios con diabetes mellitus de acuerdo con variables sociodemográficas y clínicas y evaluar el conocimiento y la actitud hacia la enfermedad. Métodos: Este es un estudio transversal con un enfoque cuantitativo, realizado con 173 usuarios con Diabetes Mellitus registrados en el Centro de Diagnóstico del Corazón y Control de la Diabetes, en la ciudad de Vitória de Santo Antão, Pernambuco. En la recopilación de datos, se utilizó un guión sistemático para obtener las variables sociodemográficas y clínicas, así como los instrumentos de recopilación: el Cuestionario de conocimiento de diabetes para evaluar el conocimiento y el Cuestionario de actitud de diabetes para evaluar la actitud. Resultados: La población se caracterizó por adultos y ancianos, con un ingreso de hasta un salario mínimo. En cuanto al conocimiento, el $65,3 \%$ de los usuarios tienen puntuaciones inferiores a ocho puntos, lo que muestra un conocimiento insatisfactorio sobre la enfermedad. En relación con las actitudes, se encontró que la mayoría (98,3\%) de los usuarios tenían puntuaciones inferiores a 70 puntos, lo que indica dificultades para hacer frente a la enfermedad. Conclusión: Se concluye que el conocimiento y la actitud aún son incipientes, surgiendo la necesidad de implementar programas de educación en salud.

Palabras clave: Diabetes Mellitus, Conocimiento, Actitud hacia la salud.

${ }^{1}$ Universidade Federal de Pernambuco (UFPE), Vitória de Santo Antão - PE. *E-mail: valesca.souza@ufpe.br

2Universidade de Pernambuco (UPE), Petrolina - PE. 


\section{INTRODUÇÃO}

O Diabetes Mellitus (DM) está entre as doenças crônicas que apresentam elevados índices de morbimortalidade e vem se destacando como sério problema de saúde pública no Brasil e no mundo, em virtude do aumento da sua prevalência, comprometimento da qualidade de vida dos indivíduos, bem como dos altos custos com tratamentos e complicações, gerando significativos danos econômicos e sociais (POLICARPO NS, et al., 2014; GOMES AR e SANTOS L, 2017).

O DM é causado por uma combinação de fatores genéticos e estilo de vida. Os principais fatores de risco para o seu desenvolvimento são: história familiar, sobrepeso, obesidade, sedentarismo, diagnóstico prévio de pré-diabetes ou diabetes mellitus gestacional, presença de hipertensão arterial e dislipidemia. A falta de controle da doença está associada ao surgimento de complicações como distúrbios microvasculares e macrovasculares que resultam em: retinopatia, nefropatia, neuropatia, doença coronariana, doença cerebrovascular e doença arterial periférica (SOCIEDADE BRASILEIRA DE DIABETES, 2016).

Segundo a Sociedade Brasileira de Diabetes (2016), atualmente estima-se que a população mundial com diabetes seja de 387 milhões e que alcance 471 milhões em 2035. Além disso, o DM apresenta-se com um quadro de hiperglicemia, consequente de falhas na secreção e/ou na ação da insulina, sendo classificado segundo a sua etiologia em DM tipo 1, DM tipo 2, outros tipos específicos de DM e DM gestacional. Há ainda duas categorias, referidas como pré-diabetes, que são a glicemia de jejum alterada e a tolerância à glicose diminuída.

O impacto do DM está especialmente relacionado ao surgimento de complicações limitantes, que interferem na realização das atividades diárias, e assim, compromete a qualidade de vida dos indivíduos. Pois, quando o DM não é tratado adequadamente, os sintomas podem se agravar e, assim, contribuir para a manifestação de outras doenças, como problemas cardíacos, visuais, acidente vascular cerebral, insuficiência renal, lesões de difícil cicatrização, dentre outras complicações (SOCIEDADE BRASILEIRA DE DIABETES, 2016; MINISTÉRIO DA SAÚDE, 2013).

Essa condição crônica impõe ao indivíduo mudanças nos padrões de comportamento diário, como a prática de atividade física e mudanças no plano alimentar, além de comprometimento com a terapêutica medicamentosa e controle glicêmico. O que muitas vezes implicam em dificuldades para aderir ao tratamento, resultando em baixas taxas de adesão ao plano terapêutico, principalmente por requerer autocuidado em longo prazo (CORTEZ DN, et al., 2015; FERNANDES BSM, et al., 2016; MAIA MA, et al., 2016).

Portanto, para o autogerenciamento do DM é importante que os indivíduos dominem um conjunto de informações necessárias para administrar sua condição de saúde, como conhecimento sobre a doença, o tratamento e as complicações. Logo, somente o conhecimento não é suficiente para promover a mudança de comportamentos, é importante que os indivíduos também dominem habilidades necessárias para o manejo da doença (ASSUNÇÃO SC, et al., 2017; SERAMIN CM, et al., 2013).

O desenvolvimento destas capacidades é proporcionado pela educação em saúde, que é uma parte fundamental do cuidado integral ao paciente com DM, sendo evidenciada como o meio de capacitação das pessoas para realizar o controle da sua doença. A educação em saúde é uma estratégia de baixo custo que favorece a aquisição de conhecimento, a adesão de atitudes positivas frente à doença, o controle metabólico e a redução das complicações agudas e crônicas, resultando na melhora da qualidade de vida (BRITO GMG, et al., 2016; GRILLO MMF, et al., 2013).

Sendo assim, os profissionais de saúde têm um papel importante na conscientização e promoção do autocuidado às pessoas com DM e seus familiares, através do fornecimento de informações que possibilitem ao usuário lidar com situações do dia a dia, advindas da doença (MARQUES MB, et al., 2013).

Desta forma, considera-se que a educação para o auto manejo do DM objetiva alcançar a melhoria da qualidade de vida dos indivíduos e que esses programas podem agregar valores positivos para a aquisição de conhecimento e habilidades, refletindo-se, consequentemente, na qualidade de vida (BRITO GMG, et al., 2016). Assim, este estudo tem por objetivo caracterizar os usuários com Diabetes Mellitus segundo as variáveis sociodemográficas e clínicas e avaliar o conhecimento e atitude em relação à doença. 


\section{MÉTODOS}

Estudo transversal de abordagem quantitativa, realizado no próprio domicílio dos usuários que estavam cadastrados no ano 2016, no Centro de Diagnósticos do Coração e Controle do Diabetes, localizado em um município do Pernambuco, Brasil. Essa unidade foi eleita pela sua área de abrangência e número de usuários com DM. A população cadastrada com DM na unidade no ano 2016 correspondeu a 367 usuários, foi feito o cálculo amostral para população finita e variável qualitativa com $95 \%$ de confiança que resultou numa amostra de 188 usuários. Esses usuários foram selecionados através de sorteio aleatório realizado pela pesquisadora.

Durante a coleta de dados ocorreram 15 perdas, no qual 3 usuários haviam falecido, 4 não residiam mais no mesmo endereço e 8 não aceitaram participar da pesquisa, resultando numa amostra final de 173 usuários que atenderam aos critérios de inclusão: usuários com idade igual ou superior a 18 anos e assistidos na unidade em 2016; e os critérios de exclusão: usuários com déficit cognitivo inviabilizando o preenchimento do questionário.

Para a coleta de dados foi utilizado roteiro sistematizado para obter as variáveis sociodemográficas: sexo, idade, escolaridade, estado civil, ocupação, renda familiar, número de membros na família; e variáveis clínicas: diagnóstico, tempo de doença e tipo de tratamento. Para a coleta dos dados referentes ao conhecimento e atitudes foram utilizados os questionários Diabetes Knowledge Questionnaire (DKN-A) e Diabetes Attitude Questionnaire (ATT-19). Esses instrumentos foram traduzidos para a língua portuguesa e validados no Brasil (TORRES HC, et al., 2005).

O DKN-A é um questionário com 15 itens de múltipla escolha acerca de diferentes tópicos relacionados ao conhecimento geral do DM. Contém cinco amplas categorias: a) fisiologia básica, incluindo a ação da insulina, b) hipoglicemia, c) grupos de alimentos e suas substituições, d) gerenciamento de DM na intercorrência de alguma outra doença, e) princípios gerais dos cuidados da doença. A escala de medida é de 0-15 e cada item é medido com escore um para resposta correta e zero para incorreta. Os itens de 1 a 12 requerem uma única resposta correta. Para os itens de 13 a 15 algumas respostas são corretas e todas devem ser conferidas para obter o escore um. Um escore maior que oito indica conhecimento sobre 0 Diabetes mellitus (TORRES HC et al., 2005).

O ATT-19 é um questionário sobre a medida de ajustamento psicológico para Diabetes mellitus, desenvolvido como resposta às necessidades de avaliação de aspectos psicológicos e emocionais sobre a doença. Consiste em 19 itens que incluem seis fatores: a) estresse associado a Diabetes mellitus, b) receptividade ao tratamento, c) confiança no tratamento, d) eficácia pessoal, e) percepção sobre a saúde, f) aceitação social. Cada resposta é medida pela escala de Likert de cinco pontos (discordo totalmente - escore 1; até concordo totalmente - escore 5). O valor total da taxa-escore varia de 19 a 95 pontos. Um escore maior que 70 pontos indica a atitude positiva sobre a doença (TORRES HC et al., 2005).

A pesquisadora, inicialmente, realizou o levantamento dos usuários e seus respectivos endereços e telefones. De posse dessas informações, dirigiu-se ao próprio domicílio dos usuários para convidá-lo a participar da pesquisa, esclarecendo a natureza e os objetivos do estudo e, após a assinatura do termo de consentimento livre e esclarecido pelo usuário, iniciou-se a entrevista. Foram aplicados os instrumentos referentes aos dados sociodemográficos e clínicos, seguidos dos questionários DKN-A e ATT-19.

Os dados foram coletados no mês de setembro de 2017. O tempo de cada entrevista foi de aproximadamente 25 minutos. Os questionários DKN-A e ATT-19 são autoaplicáveis, no entanto, os usuários apresentaram dificuldades no preenchimento e por isso o processo de coleta contou com o auxílio da pesquisadora. Para análise, foi elaborado um banco de dados no Programa Excel 2010. Os dados foram transportados para o Programa SPSS 13.0 (Statistical Package for the Social Sciences) para Windows. Todos os testes foram aplicados com $95 \%$ de confiança.

Os resultados estão apresentados em forma de tabela e gráficos com suas respectivas frequências absoluta e relativa; as variáveis numéricas estão representadas pelas medidas de tendência central e medidas de dispersão. Para verificar a diferença estatística foram utilizados o Teste Qui-Quadrado e o Teste Exato de Fisher para as variáveis categóricas; bem como o Teste de Normalidade de Kolmogorov-Smirnov para variáveis quantitativas; e para a Comparação com dois grupos: Mann-Whitney (Não Normal). 
Conforme a Resolução 466/12 do Conselho Nacional de Saúde (BRASIL, 2012), este projeto, recebeu aprovação do Comitê de Ética em Pesquisa do Centro de Ciências da Saúde da Universidade Federal de Pernambuco (UFPE) sob o CAAE: n71473517.2.0000.5208, no do parecer: 2.264.434.

\section{RESULTADOS}

A amostra foi composta por 173 pacientes, com média de idade de 59,41 $\pm 12,38$ anos e predominância do sexo feminino $(36,9 \%)$. A maioria era casado $(50,3 \%)$ e não tinham concluído e ensino fundamental (60,2\%). 54,3\% eram aposentados e $69,9 \%$ declararam renda de até um salário mínimo. A maioria dos participantes $(63,6 \%)$ não soube informar o seu tipo de diabetes. Quanto ao tratamento, $72,8 \%$ faziam uso de antidiabético oral e $75,7 \%$ referiram seguir a dieta recomendada (Tabela 1).

Tabela 1 - Características da população estudada conforme variáveis sociodemográficas e clínicas, 2017.

\begin{tabular}{|c|c|c|}
\hline Variáveis & $\mathbf{n}$ & $\%$ \\
\hline \multicolumn{3}{|l|}{ Sexo } \\
\hline Masculino & 52 & 30,1 \\
\hline Feminino & 121 & 36,9 \\
\hline \multicolumn{3}{|l|}{ Estado Civil } \\
\hline Solteiro (a) & 34 & 19,7 \\
\hline Casado (a) & 87 & 50,3 \\
\hline Divorciado (a) & 9 & 5,2 \\
\hline Mora com companheiro (a) & 3 & 1,7 \\
\hline Viúvo (a) & 40 & 23,1 \\
\hline \multicolumn{3}{|l|}{ Escolaridade } \\
\hline Não estudou/analfabeto funcional & 30 & 17,3 \\
\hline Ensino fundamental incompleto & 104 & 60,2 \\
\hline Ensino fundamental completo & 7 & 4 \\
\hline Ensino médio incompleto & 4 & 2,3 \\
\hline Ensino médio completo & 21 & 12,1 \\
\hline Ensino superior incompleto & 1 & 0,6 \\
\hline Ensino superior completo & 6 & 3,5 \\
\hline \multicolumn{3}{|l|}{ Ocupação } \\
\hline Desempregado & 7 & 4 \\
\hline Do lar & 47 & 27,2 \\
\hline Estudante & 1 & 0,6 \\
\hline Aposentado & 94 & 54,3 \\
\hline Trabalhador assalariado & 10 & 5,8 \\
\hline Empregado doméstico & 1 & 0,6 \\
\hline Produtor rural & 4 & 2,3 \\
\hline Profissional liberal autônomo & 3 & 1,7 \\
\hline Empresário & 1 & 0,6 \\
\hline Funcionário público & 3 & 1,7 \\
\hline Outro & 2 & 1,2 \\
\hline \multicolumn{3}{|l|}{ Renda } \\
\hline Até $1 \mathrm{SM}^{*}$ & 121 & 69,9 \\
\hline Mais de $1 \mathrm{SM}^{*}$ & 52 & 30,1 \\
\hline \multicolumn{3}{|l|}{ Tipo de diabetes } \\
\hline Tipo 1 & 24 & 13,9 \\
\hline Tipo 2 & 39 & 22,5 \\
\hline Não sabe informar & 110 & 63,6 \\
\hline \multicolumn{3}{|l|}{ Tratamento atual } \\
\hline Antidiabético Oral & 126 & 72,8 \\
\hline Insulina & 97 & 56,1 \\
\hline Dieta & 131 & 75,7 \\
\hline \multirow[t]{2}{*}{ Exercício Físico } & 52 & 30,1 \\
\hline & Média \pm DP & Mínimo - Máximo \\
\hline Idade (anos completos) & $59,41 \pm 12,38$ & $18,0-85,0$ \\
\hline Número de membros da família & $2,98 \pm 1,44$ & $1,0-9,0$ \\
\hline Tempo da doença & $10,93 \pm 8,55$ & $1,0-40,0$ \\
\hline
\end{tabular}

Legenda *SM vigente - $\mathrm{R} \$ 937,00$, a partir de 1ํ de Janeiro de 2017 (IBGE 2017).

Fonte: SANTOS IM, et al., 2020. 
Em relação ao conhecimento, a maioria dos usuários $(65,3 \%)$ apresentou escores inferiores a oito pontos, o que indica um conhecimento insatisfatório sobre a doença (Figura 1).

Figura 1 - Escores obtidos no questionário DKN-A em relação ao conhecimento da doença pelos usuários com DM, atendidos na unidade estudada, 2017.

Figura 1 - Conhecimento (DKN-A)

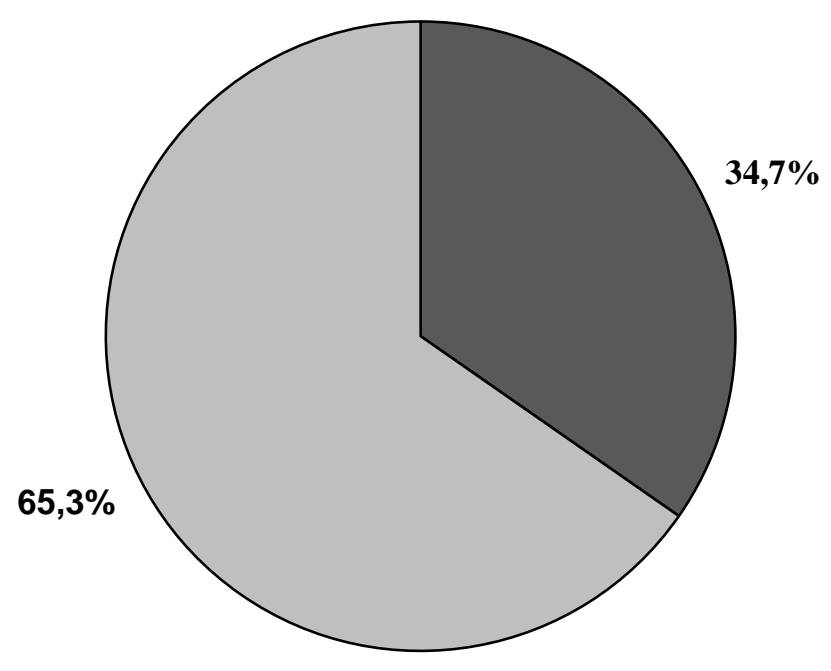

$\square$ Conhecimento satisfatório

$\square$ Conhecimento insatisfatório

Fonte: SANTOS IM, et al., 2020.

Os escores obtidos no que se refere à atitude dos usuários com DM, quando da aplicação do questionário ATT-19, constataram que a maioria apresentou escores inferiores a 70 pontos que corresponde a atitude negativa diante da doença (Figura 2).

Figura 2 - Escores obtidos no questionário ATT-19 em relação às atitudes de enfrentamento da doença pelos usuários com DM, atendidos na unidade estudada, 2017.

Figura 2 - Atitude Positiva (ATT-19)

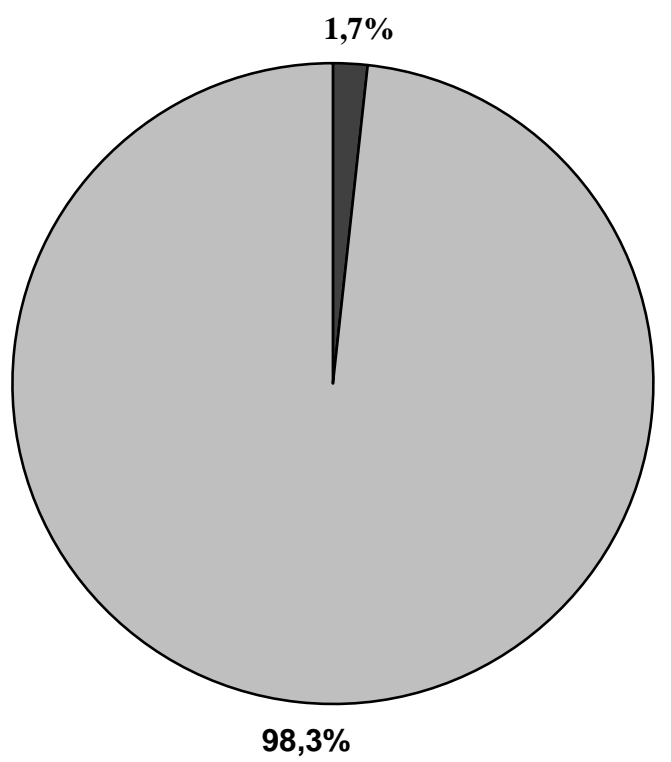

$\square$ Atitude positiva

$\square$ Atitude negativa

Fonte: SANTOS IM, et al., 2020. 
As variáveis que apresentaram relação estatisticamente significativa com o conhecimento sobre DM foram: escolaridade $(p<0,001)$, renda $(p=0,015)$, tipo de diabetes $(p=0,008)$ e uso de insulina $(p=0,041)$ (Tabela 2).

Tabela 2 - Relação entre os escores do conhecimento (DKN-A), segundo as variáveis: sexo, estado civil, escolaridade, renda, tipo de diabetes, tratamento atual e tempo de doença dos usuários com DM, atendidos na unidade estudada, 2017.

\section{Total DKN-A (Conhecimento DM)}

\begin{tabular}{|c|c|c|c|}
\hline Variáveis & Sim n (\%) & Não n (\%) & p-valor \\
\hline \multicolumn{4}{|l|}{ Sexo } \\
\hline Masculino & $19(36,5)$ & $33(63,5)$ & \multirow{2}{*}{$0,737^{*}$} \\
\hline Feminino & $41(33,9)$ & $80(66,1)$ & \\
\hline \multicolumn{4}{|l|}{ Estado Civil } \\
\hline Solteiro (a) & $16(47,1)$ & $18(52,9)$ & \multirow{5}{*}{0,383 ** } \\
\hline Casado (a) & $28(32,2)$ & $59(67,8)$ & \\
\hline Divorciado (a) & $4(44,4)$ & $5(55,6)$ & \\
\hline Mora com companheiro (a) & $1(33,3)$ & $2(66,7)$ & \\
\hline Viúvo (a) & $11(27,5)$ & $29(72,5)$ & \\
\hline \multicolumn{4}{|l|}{ Escolaridade } \\
\hline Não estudou & $6(20,0)$ & $24(80,0)$ & \multirow{7}{*}{$<0,001$ ** } \\
\hline Ensino fundamental incompleto & $28(26,9)$ & $76(73,1)$ & \\
\hline Ensino fundamental completo & $4(57,1)$ & $3(42,9)$ & \\
\hline Ensino médio incompleto & $3(75,0)$ & $1(25,0)$ & \\
\hline Ensino médio completo & $13(61,9)$ & $8(38,1)$ & \\
\hline Ensino superior incompleto & $1(100,0)$ & $0(0,0)$ & \\
\hline Ensino superior completo & $5(83,3)$ & $1(16,7)$ & \\
\hline \multicolumn{4}{|l|}{ Renda } \\
\hline Até $1 \mathrm{SM}$ & $35(28,9)$ & $86(71,1)$ & \multirow{2}{*}{$0,015^{*}$} \\
\hline Mais de $1 \mathrm{SM}$ & $25(48,1)$ & $27(51,9)$ & \\
\hline \multicolumn{4}{|l|}{ Tipo de diabetes } \\
\hline Tipo 1 & $13(54,2)$ & $11(45,8)$ & \multirow{3}{*}{0,008 * } \\
\hline Tipo 2 & $18(46,2)$ & $21(53,8)$ & \\
\hline Não sabe informar & $29(26,4)$ & $81(73,6)$ & \\
\hline \multicolumn{4}{|l|}{ Tratamento atual } \\
\hline Antidiabético Oral & $42(33,3)$ & $84(66,7)$ & 0,542 * \\
\hline Insulina & $40(41,2)$ & $57(58,8)$ & $0,041^{*}$ \\
\hline Dieta & $43(32,8)$ & $88(67,2)$ & 0,365 * \\
\hline \multirow[t]{2}{*}{ Exercício Físico } & $21(40,4)$ & $31(59,6)$ & 0,302 * \\
\hline & Média \pm DP & Média \pm DP & \\
\hline Tempo da doença & $10,23 \pm 8,92$ & $10,77 \pm 8,38$ & $0,835^{\star * *}$ \\
\hline
\end{tabular}

Legenda: $\left(^{\star}\right)$ Teste Qui-Quadrado $\left.{ }^{(*}\right)$ Teste Exato de Fisher $\left({ }^{* \star *}\right)$ Teste de Mann-Whitney.

Fonte: SANTOS IM, et al., 2020.

\section{DISCUSSÃO}

O conhecimento e a atitude para o automanejo do DM são fatores relevantes para o controle ideal da doença, pois a partir da compreensão do plano de cuidados, o indivíduo é capaz de assumir um comprometimento terapêutico e, assim obter resultados positivos no controle glicêmico (SERAMIN CM, et al., 2013; CARDOSO AF, et al., 2015).

Para a aquisição desses conhecimentos e habilidades é indispensável que os usuários estejam envolvidos no processo de educação em saúde. A educação para o automanejo do DM é o processo de ensinar o usuário a administrar a sua doença (SERAMIN CM, et al., 2013). Os programas de educação em saúde para as pessoas com DM agregam estratégias para solucionar problemas e agravos decorrentes da doença, capazes de proporcionar uma melhora do conhecimento, contribuindo para a mudança de comportamento.

A maioria dos participantes deste estudo eram do sexo feminino, semelhante ao encontrado por Maia MA, et al. (2016) ao observar uma amostra de 151 pessoas com DM, onde $76,8 \%$ era do sexo feminino. Esta 
prevalência pode ser explicada pelo fato de as mulheres terem maior tendência à procura de assistência em serviços de saúde do que os homens, o que aumenta a probabilidade de serem diagnosticadas com a doença (ROSSANEIS MA, et al., 2016). Este cenário evidencia a necessidade de implementar ações que estimulem a participação do sexo masculino nas unidades de saúde (POLICARPO NS, et al., 2014).

Quanto ao estado civil, a maioria dos usuários eram casados. Segundo um estudo realizado por Oliveira MSS, et al. (2014), pessoas casadas, tendem a apresentar melhor aderência ao tratamento, uma vez que há uma estrutura de amparo associada ao lado psíquico-emocional do paciente (OLIVEIRA MSS, et al., 2014). Enquanto a condição de morar sozinho pode ser um forte indicador para a não adesão à terapêutica, pois a vida familiar acaba por influenciar a tomada de decisão quanto ao seguimento das recomendações (NETA DSR, et al., 2015).

Em relação à escolaridade, houve predominância de usuários com baixo grau de instrução. Esses dados concordam com uma pesquisa que foi realizada no município de Montes Claros - MG, Brasil, com 353 usuários, no qual foi visto que $51,8 \%$ apresentavam baixo grau de escolaridade. Esta condição pode prejudicar a adesão ao tratamento estabelecido, devido à dificuldade em ler e compreender a conduta terapêutica, aumentando, assim, os riscos à saúde (ASSUNÇÃO SC, et al., 2017).

No presente estudo, obteve-se predomínio de aposentados, assim como apresenta um estudo elaborado no município de Bebedouro - SP, Brasil, realizado com 44 indivíduos, onde foi visto que $43,2 \%$ eram aposentados (SERAMIN CM, et al., 2013). Esse fato pode apresentar-se como uma oportunidade para os usuários modificarem a dinâmica de vida, visto que pela disponibilidade de tempo, adotam práticas mais saudáveis, como a realização de exercício físico, o que contribui para o controle da doença (BRESSAN MALC, et al., 2013).

Quanto a renda familiar, a maioria dos usuários recebiam até um salário mínimo. Deve-se considerar que fatores ligados à baixa renda, condições de vida instáveis, duração do tratamento e alto custo de medicações podem afetar negativamente a adesão ao tratamento do DM, pois o usuário precisa retirar mensalmente determinada quantia do seu orçamento para custear tais demandas, essa realidade gera impactos financeiros que refletem no enfrentamento pessoal do indivíduo quanto à doença, associando-a a uma percepção negativa (FREITAS EF, et al., 2015).

Em relação ao tipo do diabetes, a maioria dos usuários referiram não saber informar, esta condição indica uma deficiência de conhecimento sobre a doença. Assim sendo, um dos fatores que precisam ser considerados na assistência à saúde é a promoção do conhecimento, considerando que os usuários são responsáveis pelo seu próprio cuidado e bem-estar e uma definição básica sobre a patologia que o acomete, é uma informação necessária para condução adequada do tratamento (GREGHI EFM e PASCON DM, 2016).

No que tange ao tempo da doença, os usuários com DM apresentaram uma média de 10,93 anos. Um estudo realizado em Minas Gerais, Brasil, com 1.320 usuários, observou que a presença de complicações do DM pode estar associada ao tempo de duração da doença, no qual os usuários com diagnóstico há mais de 10 anos, apresentaram um percentual de complicações de $32,2 \%$ e os usuários com menos de 5 anos de diagnóstico, apresentaram um percentual de $12,1 \%$ para complicações relacionadas ao DM (CORTEZ DN, et al., 2015).

O aumento das complicações do DM ao longo dos anos e o tempo de duração da doença também não garante que o usuário tenha conhecimento sobre a patologia, muito menos, melhoria quanto à prontidão para o autocuidado. Diante disso, é importante ressaltar que o estímulo ao autocuidado por parte dos profissionais de saúde para o indivíduo diabético e seus familiares precisa ser contínuo, visto que é difícil modificar o estilo de vida e, mais ainda, manter essas modificações.

Em relação ao tratamento, a maioria dos usuários desse estudo fazem uso de medicamentos oral, insulina e referiram seguir a dieta recomendada, porém só 30,1\% dos usuários relataram realizar exercício físico, resultado semelhante ao encontrado em estudo realizado no município Viçosa, Minas Gerais, onde os dados referiram que apenas $20 \%$ dos indivíduos relataram praticar exercícios regularmente (FREITAS EF, et al., 2015). A prática de exercício físico juntamente com a adoção de hábitos alimentares saudáveis, 
correspondem a uma estratégia de intervenção não medicamentosa capaz de gerar efeitos positivos tanto na prevenção do DM, como na redução do aparecimento de complicações relacionadas à doença (FREITAS EF, et al., 2015).

Ao analisar os escores obtidos em relação ao conhecimento, obteve-se que a maioria dos usuários apresentou escores inferiores a oito pontos, o que indica um conhecimento insatisfatório sobre a doença. Estes achados concordam com o estudo realizado por Assunção SC, et al. (2017), no qual, 56,1\% dos indivíduos também alcançaram escores inferior a oito pontos, indicando baixo conhecimento sobre a doença.

Os resultados em relação ao conhecimento do DM sobre questões importantes como as complicações, os grupos de alimentos e suas substituições foram insatisfatórios. Estes achados podem estar associados a idade avançada e a baixa escolaridade. Nesse sentido, a educação sobre DM é essencial para reduzir os riscos de complicações crônicas relacionadas à doença. Os profissionais devem utilizar estratégias educacionais que possibilitem a obtenção de conhecimento por parte dos usuários para o manejo da DM e, assim, modificar suas atitudes diante da doença (SANTOS BMO, et al., 2016).

Vale ressaltar o quanto é importante para a aquisição de conhecimento o oferecimento de ações educativas nos serviços de saúde. As ações educativas são capazes de transmitir informações que ajudam o usuário na aquisição de práticas para o controle do diabetes e o seu autocuidado, além de sensibilizar o usuário sobre os fatores de risco. Mas também, é preciso considerar que nem sempre o conhecimento leva a mudança de atitude frente às situações advindas da doença (ASSUNÇÃO SC, et al., 2017).

A falta de conhecimento adequado sobre a doença pode refletir em pouca importância para o autocuidado, seja por descuido, ou mesmo, por falta de informação acerca das complicações. Furlan C, et al. (2019) demonstraram que o conhecimento deficiente está provavelmente relacionado a baixa escolaridade, visto que a maioria dos pacientes afirmam saber o que é diabetes, mas não conseguem conceituá-la adequadamente. Portanto, a responsabilidade do autocuidado só deve ser designada ao paciente após a certeza da compreensão sobre a doença e dos cuidados que ela exige.

Os escores obtidos em relação às atitudes constataram que a maioria dos usuários apresentaram escores inferiores a 70 pontos, indicando dificuldades no enfrentamento da doença. Em concordância com o estudo realizado em uma Unidade de Atenção Primária à Saúde em Montes Claros, MG, Brasil, no qual obteve que $97,7 \%$ dos participantes apresentaram escores iguais ou menores que 70 pontos, sinalizando que ainda não alcançaram atitude positiva frente às modificações necessárias para obtenção de um bom controle metabólico (ASSUNÇÃO SC, et al., 2017).

Portanto, o diabetes mellitus merece atenção especial entre as doenças crônicas não transmissíveis que os sistemas de saúde enfrentam atualmente. É indispensável que os profissionais de saúde tomem iniciativas que incentivem práticas de promoção de saúde e hábitos de vida saudáveis. Essas práticas são capazes de gerar um impacto positivo na qualidade de vida desses indivíduos e podem proporcionar melhora nas condições de saúde desta população (CARDOSO AF, et al., 2015).

A formação continuada para a autogestão da doença traz resultados positivos no controle glicêmico como na redução do risco de complicações (CARDOSO AF, et al., 2015). Assim, o contato desses usuários com os programas educativos pode proporcionar melhora do conhecimento, seguido de mudança de comportamento e, por conseguinte, melhora da qualidade de vida.

Os dados do presente estudo apontam para a necessidade de implantação de programas de educação em saúde, visando minimizar as dificuldades encontradas no dia a dia pelos usuários em relação ao conhecimento e atitudes a fim de obter um bom controle metabólico. Associado a isso, sugere-se a necessidade de os profissionais de saúde conhecerem a realidade de cada indivíduo, a fim de planejar intervenções adequadas que facilitem a compreensão das informações oferecidas, prestando um serviço de melhor qualidade à população.

O enfrentamento quanto a essa patologia crônica é delineado a partir do perfil do paciente, portanto é indispensável para realização de intervenções educativas em saúde efetivas o conhecimento apurado acerca do paciente e o ambiente que o circunda. $O$ seguimento de orientações ministeriais como a política de 
promoção à saúde e o caderno de atenção básica: estratégias para o cuidado da pessoa com doença crônica, a educação em saúde e a educação permanente, voltada aos profissionais, formam uma tríade que quando funcional potenciam a adesão ao tratamento, trazendo reflexos positivos ao controle glicêmico e à qualidade de vida (CARDOSO AF, et al., 2015).

Como limitações deste estudo, destaca-se que foi realizado em um município de pequeno porte, com indivíduos cadastrados em uma única unidade de atendimento a pessoas com DM, o que impossibilita a generalização dos dados para outros cenários e instituições. Sugere-se, então, a realização de outros estudos em municípios maiores para que se tenha dados robustos acerca da temática.

\section{CONCLUSÃO}

Os resultados deste estudo apontaram que quanto ao conhecimento da doença, a maioria dos usuários apresentou escores insatisfatórios para a compreensão e adoção de práticas de autocuidado e, quanto aos escores de atitude, apresentaram escores menores que 70 pontos, quase em sua totalidade de usuários, indicando baixa prontidão para o enfrentamento da doença. Desta forma a atitude/enfrentamento em relação a essa patologia crônica é delineado conforme o perfil do paciente. Portanto, o conhecimento aguçado acerca do paciente e os fatores ambientais que o circundam precisam ser considerados frente à formulação de intervenções educativas, que visam à melhoria na adesão ao tratamento. Espera-se que este estudo contribua para a reflexão dos profissionais de saúde em relação a importância de se atualizarem constantemente e também quanto a relevância da educação em saúde para as pessoas com Diabetes Mellitus afim de facilitar o conhecimento, o desenvolvimento de habilidades e a capacidade para o autocontrole, e, consequentemente, melhorar a saúde e a qualidade de vida dos pacientes.

\section{REFERÊNCIAS}

1. ASSUNÇÃO SC, et al. Conhecimento e atitude de pacientes com diabetes mellitus da Atenção Primária à Saúde. Escola Anna Nery, 2017; 21(4): e20170208.

2. BRASIL. Resolução № 466/12 do Conselho Nacional de Saúde, de 12 de dezembro de 2012. Aprova as diretrizes e normas regulamentadora de pesquisas envolvendo seres humanos. Diário Oficial da União, 2012.

3. BRESSAN MALC, et al. Bem-estar na aposentadoria: o que isto significa para os servidores públicos federais? Revista Brasileira de Geriatria e Gerontologia, 2013; 16(2): 259-272.

4. BRITO GMG, et al. Quality of life, knowledge and attitude after educational program for Diabetes. Acta Paulista de Enfermagem, 2016; 29(3): 298-306.

5. CARDOSO AF, et al. Intervenções para a aquisição do autocuidado terapêutico da pessoa com diabetes mellitus: revisão sistemática da literatura. Revista Portuguesa de Saúde Pública, 2015; 33(2): 246-255.

6. CORTEZ DN, et al. Complicações e o tempo de diagnóstico do diabetes mellitus na atenção primária. Acta Paulista de Enfermagem, 2015; 28(3): 250-255.

7. FERNANDES BSM, et al. Development, validation and cultural adaptation of the Compasso protocol: Adherence to self-care in diabetes. Acta Paulista de Enfermagem, 2016;29(4): 421-429.

8. FREITAS EF, et al. Prevalência de diabetes mellitus e prática de exercício em indivíduos que procuraram atendimento na estratégia saúde da família de Viçosa/MG. Revista da Educação Física/UEM, 2015; 26(4): 549-556.

9. FURLAN C, et al. Nível de conhecimento sobre diabetes e perfil epidemiológico dos pacientes diabéticos atendidos no Hospital Electro Bonini de Ribeirão Preto/SP. Semina: Ciências Biológicas e da Saúde, Londrina, jul./dez. 2019; 40(2): 197-202.

10. GOMES AR, SANTOS L. Prevalência das complicações da diabetes mellitus no ACeS Santo Tirso/Trofa: estudo descritivo. Revista Portuguesa de Medicina Geral e Familiar, 2017; 33(4): 252-260.

11. GREGHI EFM, Pascon DM. Conhecimento dos pacientes portadores de diabetes mellitus tipo 2 inseridos no programa de saúde ao adulto. Revista da Faculdade de Ciências Médicas de Sorocaba, 2016; 18(4): 204-209.

12. GRILLO MFF, et al. Effect of different types of self-management education in patients with diabetes. Revista da Associação Médica Brasileira, 2013; 59(4): 400-405.

13. MAIA MA, et al. Associação do tempo de contato no programa educativo em diabetes mellitus

14. no conhecimento e habilidades de autocuidado. Revista Escola de Enfermagem da USP, 2016; 50(1): 59-65.

15. MARQUES MB, et al Avaliação da competência de idosos diabéticos para o autocuidado. Revista da Escola de Enfermagem da USP, 2013; 47(2): 4415-4420;

16. MINISTÉRIO DA SAÚDE. Secretaria de Atenção à Saúde, Departamento de Atenção Básica. Estratégias para o cuidado da pessoa com doença crônica: diabetes mellitus. Brasília: Ministério da Saúde, 2013.

17. NETA DSR, et al. Adesão das pessoas com diabetes mellitus ao autocuidado com os pés. Revista Brasileira de Enfermagem, 2015; 68(1): 111-116.

18. OLIVEIRA MSS, et al. Avaliação da adesão terapêutica de pacientes com diabetes mellitus tipo 2 . Revista de Enfermagem UFPE online, 2014; 8(6): 1692-1701. 
19. POLICARPO NS, et al. Knowledge, attitudes and practices for the prevention of diabetic foot. Revista Gaúcha de Enfermagem, 2014; 35(3): 36-42.

20. ROSSANEIS MA, et al. Diferenças entre mulheres e homens diabéticos no autocuidado com os pés e estilo de vida. Revista Latino-Americana de Enfermagem, 2016; 24(1): e2761.

21. SANTOS BMO, et al. Conhecimento e atitudes em diabetes mellitus tipo 2: subsídios para autocuidado e promoção de saúde. Arq. Ciênc. Saúde. 2016 out-dez; 23(4): 31-36

22. SERAMIN CM, et al. Conhecimento e atitude: componentes para a educação em diabetes mellitus nas unidades básicas de saúde de Bebedouro, SP. Revista Fafibe On-Line, 2013; 1(1): 130-139.

23. SOCIEDADE BRASILEIRA DE DIABETES (SBD). Diretrizes da Sociedade Brasileira de Diabetes (2015-2016). São Paulo: AC Farmacêutica, 2016.

24. TORRES HC, et al. Validação dos questionários de conhecimento (DKN-A) e atitude (ATT-19) de Diabetes Mellitus. Revista de Saúde Pública, 2005; 39(6): 906-911. 